\title{
Integrable flows between exact CFTs
}

\section{George Georgiou $^{a}$ and Konstantinos Sfetsos ${ }^{b, c}$}

\author{
${ }^{a}$ Institute of Nuclear and Particle Physics, National Center for Scientific Research Demokritos, \\ Ag. Paraskevi, GR-15310 Athens, Greece \\ ${ }^{b}$ Department of Nuclear and Particle Physics, Faculty of Physics, \\ National and Kapodistrian University of Athens, \\ Athens 15784, Greece \\ ${ }^{c}$ Theoretical Physics Department, CERN, \\ CH-1211 Geneva 23, Switzerland \\ E-mail: georgiou@inp.demokritos.gr, ksfetsos@phys.uoa.gr
}

ABSTRACT: We explicitly construct families of integrable $\sigma$-model actions smoothly interpolating between exact CFTs. In the ultraviolet the theory is the direct product of two current algebras at levels $k_{1}$ and $k_{2}$. In the infrared and for the case of two deformation matrices the CFT involves a coset CFT, whereas for a single matrix deformation it is given by the ultraviolet direct product theories but at levels $k_{1}$ and $k_{2}-k_{1}$. For isotropic deformations we demonstrate integrability. In this case we also compute the exact beta-function for the deformation parameters using gravitational methods. This is shown to coincide with previous results obtained using perturbation theory and non-perturbative symmetries.

Keywords: Conformal Field Models in String Theory, Conformal Field Theory, Integrable Field Theories, Sigma Models

ARXIV EPRINT: 1707.05149 


\section{Contents}

1 Introduction 1

2 Constructing the Lagrangian $\quad 3$

2.1 Two limits 6

2.1.1 One vanishing deformation matrix 6

$\begin{array}{lll}2.1 .2 & \text { Zooming in } & 7\end{array}$

$\begin{array}{lll}3 & \text { Integrability } & 7\end{array}$

$\begin{array}{ll}3.1 & \text { Lax pairs and charges in involution } \\ \end{array}$

4 The $\beta$-function $\quad 10$

5 Symmetry at the IR conformal point $\quad 13$

$\begin{array}{lll}\text { 5.1 CFT and its symmetries at } \lambda_{1}=\lambda_{2}=\lambda_{0} & 14\end{array}$

$\begin{array}{lll}5.2 \text { CFT and its symmetries at } \lambda_{1}=\lambda_{0}, \lambda_{2}=0 & 15\end{array}$

6 Discussions and future directions $\quad 16$

\section{Introduction}

In recent years all-loop effective actions describing various deformations of current algebra conformal field theories (CFTs) have been constructed. These deformed theories posess novel non-perturbative in the deformation parameters quantum symmetries which are classically realized by the effective actions. They also provide new integrable $\sigma$-models and serve as the starting point for constructing new type-II supergravity solutions.

The prototype example for these developments has been a deformation of the WZW action $S_{k}(g)$, where $g$ is an element of a general semi-simple group $G$. The WZW action perturbed by a current bilinear is given by

$$
S=S_{k}(g)+\frac{k}{\pi} \int d^{2} \sigma \lambda^{a b} J_{+}^{a} J_{-}^{b} .
$$

In the absence of the bilinear term, the $J_{+}^{a}$ 's and the $J_{-}^{b}$ 's obey two commuting current algebras both at the same positive level $k$. The coupling constants $\lambda_{a b}$, where $a, b=$ $1,2, \ldots, \operatorname{dim} G$, are elements of a matrix $\lambda$. Due to the deformation, the original WZW model is driven towards the infrared (IR) away from the conformal point which is in the ultraviolet (UV).

The effective action for (1.1) which takes into account all loops in $\lambda$ but is valid for large $k$, was constructed in [1] and for $\lambda_{a b}=\lambda \delta_{a b}$ was shown to correspond to an integrable $\sigma$-model. These models are generically called $\lambda$-deformed. These considerations 
were extended to the case where the WZW model is replaced by a coset CFT [1-3], as well as to the case of supergroups $[2,3]$. The computation of the renormalization group (RG) flow equations that are exact in $\lambda$ but for large $k$ using gravitational methods was performed in $[4,5]$. The results agree with those obtained from field theoretical methods in the past $[6$, 7] and more recently in [8]. Furthermore, deformed models of low dimensionality have been embedded to supergravity [9-13]. Integrability has also been shown for $\lambda$-deformed models corresponding to symmetric coset spaces $[2,3]$, for the $\mathrm{SU}(2)$ group case and diagonal matrix $\lambda[14]$, and for the models in [15]. Moreover, the $\lambda$-deformations were shown to be related via Poisson-Lie T-duality [22] and appropriate analytic continuations [15, 23$26]$ to a different type of integrable deformations, the so-called $\eta$-deformations for group and coset spaces introduced in [16-18] and [19-21], respectively. For the case of isotropic couplings, i.e. $\lambda_{a b}=\lambda \delta_{a b}$ and for $k \gg 1$, all-loop correlators of current and primary field operators have been computed in $[27,28]$. In these computations a few terms obtained using perturbation theory and the non-perturbative symmetry, argued via path integral considerations in [29], were enough to obtain the exact results. Other selected and related recent works can be found in [30-40].

One may wonder in which sense the action constructed in [1] is the unique all-loop in $\lambda$ effective action for (1.1). There are several facts pointing towards that intepretation. First of all, both actions have the same global symmetries and coincide up to $\mathcal{O}(\lambda)$. Moreover, the action of [1] realizes classically the quantum symmetries of (1.1) and it correctly reproduces the all-loop beta-functions and anomalous dimensions of the currents. Finally, the exact in $\lambda$ operator product expansion (OPE) of the currents was computed and the corresponding Poisson brackets were extracted [27] (see section 6). These were shown to coincide with the bracket algebra of [41] which is realized by the action of [1].

The above developments make it apparent that it is worth to pursue further this line of research. Consider the following modification of (1.1)

$$
S=S_{C F T}+\frac{\sqrt{k_{1} k_{2}}}{\pi} \int d^{2} \sigma \lambda^{a b} J_{+}^{a} J_{-}^{b},
$$

where $S_{C F T}$ is the action of a CFT possessing left and right conserved currents $J_{+}^{a}$ and $J_{-}^{a}$ which obey the standard Kac-Moody algebras with levels $k_{1}$ and $k_{2}$, respectively. This theory was studied in [42] where the beta-functions of the model were evaluated. Subsequently, the authors of [43] computed the exact anomalous dimensions of current and primary field operators using CFT perturbation theory and non-perturbative quantum symmetries argued in [29]. The essential feature of the model in (1.2) is that under the $\mathrm{RG}$ flow a new fixed point in the IR is reached. This fixed point is not present in the case where the levels are equal. This feature is very appealing but at the same time the theory is chiral due to the levels being unequal. This fact makes the Lagrangian description of the theory, not to mention the construction of an effective all loop action, an important and highly non-trivial task which remained elusive until the present paper. 
In a parallel development the all-loop effective action of two WZW actions for the group elements $g_{1}, g_{2} \in G$ mutually interacting via current bilinears, i.e.

$$
S=S_{k}\left(g_{1}\right)+S_{k}\left(g_{2}\right)+\frac{k}{\pi} \int d^{2} \sigma\left(\left(\lambda_{1}\right)^{a b} J_{1+}^{a} J_{2-}^{b}+\left(\lambda_{2}\right)^{a b} J_{2+}^{a} J_{1-}^{b}\right)
$$

was constructed in [44]. In this model there are four commuting current algebras generated by the $J_{1+}^{a}$ 's, $J_{2+}^{a}$ 's as well by the $J_{1-}^{a}$ 's, $J_{2-}^{a}$ 's, all at level $k$. The current bilinear terms above represent mutual interactions between the two WZW models. Self-interacting terms of the form appearing in (1.1) and (1.2) are absent. The anomalous dimensions of current and primaries in this theory were computed using CFT techniques and symmetry arguments in [45]. It turns out that the effective action corresponding to (1.3) is canonically equivalent [46] to the effective action of the sum of two models of the form (1.1). As such the beta-functions for the couplings are identical and the anomalous dimensions of operators are related.

The purpose of the present paper is to find an action realizing all loop effects of the theory (1.2). We will show that this will be provided by a modification of the procedure in [44] that led to the effective action for (1.3).

Our construction utilizes two group elements of a general semisimple group and may have two or one distinct deformation matrices. The $\sigma$-models that we will construct are integrable and smoothly interpolate between exact CFTs. At the UV point the theory is described by the sum of two WZW models one at level $k_{1}$ and the other at level $k_{2}$. As soon as the perturbation is turned on our models are driven towards another fixed point in the IR. When both couplings are non-zero the IR CFT is described by a coset CFT the precise nature of which will be analysed in section 5 . When one of the couplings has been set to zero the IR CFT is given by the sum of two WZW models one at level $k_{1}$ and the other at level $k_{2}-k_{1}$. In both cases the flow respects Zamolodchikov's $c$-theorem. For isotropic deformations we demonstrate that the theory is integrable. We explicitly construct the Lax pairs and show that the conserved charges are in involution. We then proceed to compute the exact beta-function for the deformation parameters using gravitational methods. This is shown to coincide with previous results obtained using perturbation theory and nonperturbative symmetries of the theory [42, 43]. Finally, we present our conclusions as well as some future research directions.

\section{Constructing the Lagrangian}

In order to make the line of reasoning transparent, we first briefly review the integrable models constructed in [44] since it is a modification of these models that will give the Lagrangian we are after. Nevertheless, the reader may skip this part and jump directly to the proposed action (2.6) which we subsequently actually use. The basic idea was to generalize the construction of the $\lambda$-deformed models of [1], by first replacing the usual gauged WZW action by the following left-right asymmetric gauged action for a general 
semisimple group $G[47]$

$$
\begin{aligned}
S_{k}\left(g, A_{ \pm}, B_{ \pm}\right)=S_{k}(g)+\frac{k}{\pi} \int d^{2} \sigma \operatorname{Tr} & \left(A_{-} \partial_{+} g g^{-1}-B_{+} g^{-1} \partial_{-} g+A_{-} g B_{+} g^{-1}\right. \\
& \left.-\frac{1}{2} A_{-} A_{+}-\frac{1}{2} B_{+} B_{-}\right)
\end{aligned}
$$

where $S_{k}(g)$ is the WZW action for the group element $g \in G$. Note also the use of two different gauge fields $A_{ \pm}$and $B_{ \pm}$taking values in the corresponding Lie algebra. Under the infinitesimal gauge transformations

$$
\delta g=g u_{R}-u_{L} g, \quad \delta A_{ \pm}=-\partial_{ \pm} u_{L}+\left[A_{ \pm}, u_{L}\right], \quad \delta B_{ \pm}=-\partial_{ \pm} u_{R}+\left[B_{ \pm}, u_{R}\right]
$$

which have different infinitesimal parameters for the left and the right transformations, the action (2.1) changes as

$$
\delta S_{k}\left(g, A_{ \pm}, B_{ \pm}\right)=\frac{k}{2 \pi} \int d^{2} \sigma \operatorname{Tr}\left[\left(A_{+} \partial_{-} u_{L}-A_{-} \partial_{+} u_{L}\right)-\left(B_{+} \partial_{-} u_{R}-B_{-} \partial_{+} u_{R}\right)\right] .
$$

This is independent of the group element. The strategy of [44] was to combine two of the aforementioned actions with two gauged PCMs as follows

$$
\begin{aligned}
S_{k}\left(g_{1}, g_{2}, \tilde{g}_{1}, \tilde{g}_{2}, A_{ \pm}, B_{ \pm}\right)= & S_{k}\left(g_{1}, A_{ \pm}, B_{ \pm}\right)+S_{k}\left(g_{2}, B_{ \pm}, A_{ \pm}\right) \\
& -\frac{1}{\pi} \int d^{2} \sigma \operatorname{Tr}\left(t^{a} \tilde{g}_{1}^{-1} D_{+} \tilde{g}_{1}\right) E_{1 a b} \operatorname{Tr}\left(t^{b} \tilde{g}_{1}^{-1} D_{-} \tilde{g}_{1}\right) \\
& -\frac{1}{\pi} \int d^{2} \sigma \operatorname{Tr}\left(t^{a} \tilde{g}_{2}^{-1} D_{+} \tilde{g}_{2}\right) E_{2 a b} \operatorname{Tr}\left(t^{b} \tilde{g}_{2}^{-1} D_{-} \tilde{g}_{2}\right)
\end{aligned}
$$

Note that the role of $A_{ \pm}$and $B_{ \pm}$is exchanged in the two gauged WZW actions. The covariant derivatives acting on the group elements defining the PCMs are $D_{ \pm} \tilde{g}_{1}=\partial_{ \pm} \tilde{g}_{1}-$ $A_{ \pm} \tilde{g}_{1}$ and $D_{ \pm} \tilde{g}_{2}=\partial_{ \pm} \tilde{g}_{2}-B_{ \pm} \tilde{g}_{2}$. The matrices $E_{1}$ and $E_{2}$ parametrize the corresponding couplings.

The virtue of this action is that it is invariant under the set of transformations

$$
\begin{aligned}
\delta g_{1} & =g_{1} u_{R}-u_{L} g_{1}, & \delta g_{2} & =g_{2} u_{L}-u_{R} g_{2}, \\
\delta \tilde{g}_{1} & =-u_{L} \tilde{g}_{1}, & \delta g_{2} & =-u_{R} \tilde{g}_{2}, \\
\delta A_{ \pm} & =-\partial_{ \pm} u_{L}+\left[A_{ \pm}, u_{L}\right], & \delta B_{ \pm} & =-\partial_{ \pm} u_{R}+\left[B_{ \pm}, u_{R}\right] .
\end{aligned}
$$

Indeed, in the first line the variation of the first term in (2.4) cancels that of the second term. The second and third lines involving the PCMs are invariant by themselves. The next step taken was to completely fix the gauge by choosing $\tilde{g}_{1}=\tilde{g}_{2}=\mathbb{I}$. The resulting gauge fixed action was given by

$$
\begin{aligned}
S\left(g_{1}, g_{2}, A_{ \pm}, B_{ \pm}\right)= & S_{k_{1}}\left(g_{1}\right)+S_{k_{2}}\left(g_{2}\right)-\frac{\sqrt{k_{1} k_{2}}}{\pi} \int d^{2} \sigma \operatorname{Tr}\left(A_{+} \lambda_{1}^{-1} A_{-}+B_{+} \lambda_{2}^{-1} B_{-}\right) \\
& +\frac{k_{1}}{\pi} \int d^{2} \sigma \operatorname{Tr}\left(A_{-} \partial_{+} g_{1} g_{1}^{-1}-B_{+} g_{1}^{-1} \partial_{-} g_{1}+A_{-} g_{1} B_{+} g_{1}^{-1}\right) \\
& +\frac{k_{2}}{\pi} \int d^{2} \sigma \operatorname{Tr}\left(B_{-} \partial_{+} g_{2} g_{2}^{-1}-A_{+} g_{2}^{-1} \partial_{-} g_{2}+B_{-} g_{2} A_{+} g_{2}^{-1}\right),
\end{aligned}
$$


where we have introduced the parameters

$$
\lambda_{i}=\sqrt{k_{1} k_{2}}\left(k \mathbb{I}+E_{i}\right)^{-1}, \quad i=1,2, \quad k=\frac{k_{1}+k_{2}}{2} .
$$

To be precise the action obtained in [44] is the one presented above but with $k_{1}=k_{2}$. Nevertheless, in (2.6) we have relaxed the condition that the two asymmetrically gauged WZW models must have the same level. We postulate this action to be our starting point. In what follows we will see that this modification drastically changes the all-loop $\beta$-functions of the model which acquire new fixed points in the IR under the flow of the renormalization group. This fact will be verified by using the gravity background generated by the all-loop effective action (2.6) which is obtained after integrating out the gauge fields $A_{ \pm}$and $B_{ \pm}$. Our results will be in complete agreement with results obtained employing CFT methods and symmetry considerations in [42] and in [43].

Integrating out the gauge fields in the action (2.6) we find that

$$
\begin{aligned}
& A_{+}=i\left(\mathbb{I}-\lambda_{1}^{T} D_{1} \lambda_{2}^{T} D_{2}\right)^{-1} \lambda_{1}^{T}\left(\lambda_{0} J_{1+}+D_{1} \lambda_{2}^{T} J_{2+}\right), \\
& A_{-}=-i\left(\mathbb{I}-\lambda_{1} D_{2}^{T} \lambda_{2} D_{1}^{T}\right)^{-1} \lambda_{1}\left(\lambda_{0}^{-1} J_{2-}+D_{2}^{T} \lambda_{2} J_{1-}\right)
\end{aligned}
$$

and that

$$
\begin{aligned}
& B_{+}=i\left(\mathbb{I}-\lambda_{2}^{T} D_{2} \lambda_{1}^{T} D_{1}\right)^{-1} \lambda_{2}^{T}\left(\lambda_{0}^{-1} J_{2+}+D_{2} \lambda_{1}^{T} J_{1+}\right), \\
& B_{-}=-i\left(\mathbb{I}-\lambda_{2} D_{1}^{T} \lambda_{1} D_{2}^{T}\right)^{-1} \lambda_{2}\left(\lambda_{0} J_{1-}+D_{1}^{T} \lambda_{1} J_{2-}\right) .
\end{aligned}
$$

The matrices $D_{a b}$ and the currents $J_{ \pm}^{a}$ are defined as

$$
J_{+}^{a}=-i \operatorname{Tr}\left(t^{a} \partial_{+} g g^{-1}\right), \quad J_{-}^{a}=-i \operatorname{Tr}\left(t^{a} g^{-1} \partial_{-} g\right), \quad D_{a b}=\operatorname{Tr}\left(t_{a} g t_{b} g^{-1}\right),
$$

where the $t^{a}$ 's are Hermitian matrices. When a current or the matrix $D$ has an index 1 or 2 this means that one should use the corresponding group element in its definition. In addition, we have defined the ratio of the two levels

$$
\lambda_{0}=\sqrt{\frac{k_{1}}{k_{2}}},
$$

which with no loss of generality can be taken to be less than one.

The substitution of the expressions for the gauge fields into the action results in a $\sigma$-model action which can be written in matrix notation as

$$
\begin{aligned}
S_{k_{1}, k_{2}, \lambda_{1}, \lambda_{2}}\left(g_{1}, g_{2}\right)= & S_{k_{1}}\left(g_{1}\right)+S_{k_{2}}\left(g_{2}\right) \\
& +\frac{1}{\pi} \int d^{2} \sigma\left(J_{1+} J_{2+}\right)\left(\begin{array}{cc}
k_{1} \Lambda_{21} \lambda_{1} D_{2}^{T} \lambda_{2} & k_{2} \lambda_{0} \Lambda_{21} \lambda_{1} \\
k_{1} \lambda_{0}^{-1} \Lambda_{12} \lambda_{2} & k_{2} \Lambda_{12} \lambda_{2} D_{1}^{T} \lambda_{1}
\end{array}\right)\left(\begin{array}{l}
J_{1-} \\
J_{2-}
\end{array}\right),
\end{aligned}
$$

where we have also defined the matrices

$$
\Lambda_{12}=\left(\mathbb{I}-\lambda_{2} D_{1}^{T} \lambda_{1} D_{2}^{T}\right)^{-1}, \quad \Lambda_{21}=\left(\mathbb{I}-\lambda_{1} D_{2}^{T} \lambda_{2} D_{1}^{T}\right)^{-1} .
$$

The above action is by construction symmetric under the exchange of the two original models, i.e. indices 1 and 2. More importantly, the model with equal levels constructed 
in [44] inherits a remarkable duality-type symmetry to the present model (2.12). This symmetry reads

$$
k_{1} \rightarrow-k_{2} \quad k_{2} \rightarrow-k_{1}, \quad \lambda_{1} \rightarrow \lambda_{1}^{-1}, \quad \lambda_{2} \rightarrow \lambda_{2}^{-1}, \quad g_{1} \rightarrow g_{2}^{-1} \quad g_{2} \rightarrow g_{1}^{-1} .
$$

The proof uses the fact that under (2.14)

$$
\begin{array}{lll}
D_{1} \rightarrow D_{2}^{T}, & J_{1+} \rightarrow-D_{2}^{T} J_{2+}, & J_{1-} \rightarrow-D_{2} J_{2-}, \\
D_{1} \rightarrow D_{2}^{T}, & J_{2+} \rightarrow-D_{1}^{T} J_{1+}, & J_{2-} \rightarrow-D_{1} J_{1-} .
\end{array}
$$

The action (2.12) may have additional global isometries for specific choices of the deformation matrices. In particular, if the $\lambda_{i}$ 's are proportional to the identity the action has the global symmetry

$$
g_{1} \rightarrow \Lambda_{L}^{-1} g_{1} \Lambda_{R}, \quad g_{2} \rightarrow \Lambda_{R}^{-1} g_{2} \Lambda_{L}, \quad \Lambda_{L}, \Lambda_{R} \in G .
$$

For general deformation matrices this symmetry is partially or completely broken.

For small elements of the matrices $\lambda_{i}$ 's the action (2.12) can be approximated by

$$
S_{k_{1}, k_{2}, \lambda_{1}, \lambda_{2}}\left(g_{1}, g_{2}\right)=S_{k_{1}}\left(g_{1}\right)+S_{k_{2}}\left(g_{2}\right)+\frac{\sqrt{k_{1} k_{2}}}{\pi} \int d^{2} \sigma\left(\lambda_{1}^{a b} J_{1+}^{a} J_{2-}^{b}+\lambda_{2}^{a b} J_{2+}^{a} J_{1-}^{b}\right)+\cdots
$$

It represents a current-current interaction of the two original WZW actions, one at level $k_{1}$ and the other at level $k_{2}$. In fact, the action (2.12) can be considered as the effective action for the theory (2.17) that incorporates all-loop effects in the deformation parameter $\lambda_{i}$.

At this point let us argue that although the theory (2.17) is left-right symmetric it reproduces correctly the all-loop correlation functions of the chiral model of (1.2). The argument goes as the one in [45]. All correlation functions involving the operators of the set $\mathcal{O}=\left\{J_{1+}^{a}, J_{2-}^{a}, J_{1+}^{a} J_{2-}^{b}, \cdots\right\}$ or any other composite operator built from them can be calculated as if the vertex proportional to $\lambda_{2}$ in (2.17) was absent. This is so because the OPE of the currents appearing in the first interaction vertex of (2.17) with any of the currents appearing in the second interaction vertex of (2.17) is regular. This means that if one restricts himself to the set of operators $\mathcal{O}$ it is as if he effectively sets $\lambda_{2}$ to zero. Thus, not only the $\beta$-functions but also all current correlation functions of the models (2.17) and (1.2) coincide to all-orders in the $\lambda$-, as well as in the $k$-expansion.

\section{$2.1 \quad$ Two limits}

\subsubsection{One vanishing deformation matrix}

Let one of the deformation parameters in (2.12) approach zero, say $\lambda_{2} \rightarrow 0$ and rename $\lambda_{1}$ to $\lambda$. Then the action (2.12) simplifies drastically to the following form

$$
S_{k_{1}, k_{2}, \lambda}\left(g_{1}, g_{2}\right)=S_{k_{1}}\left(g_{1}\right)+S_{k_{2}}\left(g_{2}\right)+\frac{\sqrt{k_{1} k_{2}}}{\pi} \int d^{2} \sigma \lambda_{a b} J_{1+}^{a} J_{2-}^{b},
$$

making the perturbative expression (2.17) exact. Note that this special case, but with $k_{1}=k_{2}$, has been examined before in $[48,49]$. Since the currents $J_{2+}^{a}$ and $J_{1-}^{a}$ do not appear 
in the action they do not acquire anomalous dimensions. This fact implies that (2.18) should have on-shell chiral and anti-chiral currents. Following a procedure parallel to that in [45] we find that the equations of motion from varying the groups elements can be cast in the form

$$
\begin{aligned}
& \partial_{-} \mathcal{J}_{+}=0, \quad \mathcal{J}_{+}=\lambda_{0}^{-1} J_{2+}+D_{2} \lambda^{T} J_{1+}, \\
& \partial_{+} \mathcal{J}_{-}=0, \quad \mathcal{J}_{-}=\lambda_{0} J_{1-}+D_{1}^{T} \lambda J_{2-} .
\end{aligned}
$$

To prove the above equations we have used the identities $\left(D^{T} \partial_{-} D\right)^{a b}=f^{a b}{ }_{c} J_{-}^{c}$ and $\left(\partial_{+} D D^{T}\right)^{a b}=f^{a b}{ }_{c} J_{+}^{c}$. The above chiral and anti-chiral conserved currents $\mathcal{J}_{ \pm}$are deformations of $J_{2+}$ and $J_{1-}$ to which they reduce for $\lambda=0$. This is consistent with their vanishing anomalous dimensions.

\subsubsection{Zooming in}

A second interesting limiting case involves taking one the group elements to unity and at the same time the corresponding level to infinity. Specifically, choosing $g_{2}$ as the relevant group element, we have that

$$
g_{2}=\mathbb{I}+i \lambda_{0} v_{a} t^{a}+\ldots, \quad k_{2} \rightarrow \infty .
$$

Hence in this limit the parameter $\lambda_{0} \rightarrow 0$. We also drop the subsctript from $g_{1}$ and the level $k_{1}$. In this limit the action (2.12) simplifies to

$$
\begin{aligned}
S_{k, \lambda_{1}, \lambda_{2}}(g, v)= & S_{k}(g)+\frac{k}{2 \pi} \int d^{2} \sigma \partial_{+} v^{a} \partial_{-} v^{a} \\
& +\frac{k}{\pi} \int d^{2} \sigma\left(J_{+} \partial_{+} v\right)\left(\begin{array}{cc}
\tilde{\Lambda}_{21} \lambda_{1} \lambda_{2} & \tilde{\Lambda}_{21} \lambda_{1} \\
\tilde{\Lambda}_{12} \lambda_{2} & \tilde{\Lambda}_{12} \lambda_{2} D^{T} \lambda_{1}
\end{array}\right)\left(\begin{array}{c}
J_{-} \\
\partial_{-} v
\end{array}\right),
\end{aligned}
$$

where

$$
\tilde{\Lambda}_{12}=\left(\mathbb{I}-\lambda_{2} D^{T} \lambda_{1}\right)^{-1}, \quad \tilde{\Lambda}_{21}=\left(\mathbb{I}-\lambda_{1} \lambda_{2} D^{T}\right)^{-1} .
$$

Note that the limit (2.20), makes the current algebras generated by $J_{2+}$ and $J_{2-}$ Abelian. Therefore, the action (2.21) represents the effective action for the mutual interaction of a WZW model at level $k$ with an Abelian theory of equal dimensionality.

\section{$3 \quad$ Integrability}

In this section, we prove that the $\sigma$-model action (2.12) is integrable when the matrices $\lambda_{1}$ and $\lambda_{2}$ are proportional to the identity, that is when $\left(\lambda_{1}\right)_{a b}=\lambda_{1} \delta_{a b}$ and $\left(\lambda_{2}\right)_{a b}=\lambda_{2} \delta_{a b}$. For the case of equal levels integrability has been shown in [44, 46]. It is remarkable that it is preserved for unequal levels as well.

\subsection{Lax pairs and charges in involution}

The integrability of (2.12) is more conveniently examined if one chooses to work with the action (2.6) before integrating out the gauge fields. 
Varying (2.6) with respect to $B_{ \pm}$and $A_{ \pm}$we find the following constraints

$$
D_{+} g_{1} g_{1}^{-1}=\left(\lambda_{0}^{-1} \lambda_{1}^{-T}-1\right) A_{+}, \quad g_{2}^{-1} D_{-} g_{2}=-\left(\lambda_{0} \lambda_{1}^{-1}-1\right) A_{-}
$$

and

$$
D_{+} g_{2} g_{2}^{-1}=\left(\lambda_{0} \lambda_{2}^{-T}-1\right) B_{+}, \quad g_{1}^{-1} D_{-} g_{1}=-\left(\lambda_{0}^{-1} \lambda_{2}^{-1}-1\right) B_{-},
$$

respectively. Varying the action with respect to group elements $g_{1}$ and $g_{2}$ results into

$$
D_{-}\left(D_{+} g_{1} g_{1}^{-1}\right)=F_{+-}^{(A)}, \quad D_{-}\left(D_{+} g_{2} g_{2}^{-1}\right)=F_{+-}^{(B)},
$$

where

$$
F_{+-}^{(A)}=\partial_{+} A_{-}-\partial_{-} A_{+}-\left[A_{+}, A_{-}\right], \quad F_{+-}^{(B)}=\partial_{+} B_{-}-\partial_{-} B_{+}-\left[B_{+}, B_{-}\right] .
$$

Equivalently, equations (3.3) can be written as

$$
D_{+}\left(g_{1}^{-1} D_{-} g_{1}\right)=F_{+-}^{(B)}, \quad D_{+}\left(g_{2}^{-1} D_{-} g_{2}\right)=F_{+-}^{(A)} .
$$

The definitions of the covariant derivatives depend on the transformation properties of the object on which they act. For example, the action on the group element $g_{1}$ involves both the $A_{ \pm}$and the $B_{ \pm}$gauge fields, that is $D_{ \pm} g_{1}=\partial_{ \pm} g_{1}-A_{ \pm} g_{1}+g_{1} B_{ \pm}$, while $D_{-}\left(D_{+} g_{1} g_{1}^{-1}\right)=$ $\partial_{-}\left(D_{+} g_{1} g_{1}^{-1}\right)-\left[A_{-},\left(D_{+} g_{1} g_{1}^{-1}\right)\right]$ involves only the $A_{ \pm}$. The next step is to substitute the constraint equations in (3.3) and (3.5). After some algebra one obtains that

$$
\begin{aligned}
\partial_{+} A_{-}-\lambda_{0}^{-1} \lambda_{1}^{-T} \partial_{-} A_{+} & =\lambda_{0}^{-1}\left[\lambda_{1}^{-T} A_{+}, A_{-}\right], \\
\lambda_{0} \lambda_{1}^{-1} \partial_{+} A_{-}-\partial_{-} A_{+} & =\lambda_{0}\left[A_{+}, \lambda_{1}^{-1} A_{-}\right]
\end{aligned}
$$

and that

$$
\begin{aligned}
\partial_{+} B_{-}-\lambda_{0} \lambda_{2}^{-T} \partial_{-} B_{+} & =\lambda_{0}\left[\lambda_{2}^{-T} B_{+}, B_{-}\right], \\
\lambda_{0}^{-1} \lambda_{2}^{-1} \partial_{+} B_{-}-\partial_{-} B_{+} & =\lambda_{0}^{-1}\left[B_{+}, \lambda_{2}^{-1} B_{-}\right] .
\end{aligned}
$$

We conclude that the equations of motion seemingly decouple forming two independent sets. Nevertheless, the fields $A_{ \pm}$and $B_{ \pm}$depend on both $\left(g_{1}, \lambda_{1}\right)$ and $\left(g_{2}, \lambda_{2}\right)$.

For the special case where $\left(\lambda_{1}\right)_{a b}=\lambda_{1} \delta_{a b}$ and $\left(\lambda_{2}\right)_{a b}=\lambda_{2} \delta_{a b}$ equations (3.6) and (3.7) can be rewritten in the form

$$
\partial_{-} A_{+}=-\frac{1-\lambda_{0} \lambda_{1}}{1-\lambda_{1}^{2}}\left[A_{+}, A_{-}\right], \quad \partial_{+} A_{-}=\frac{1-\lambda_{0}^{-1} \lambda_{1}}{1-\lambda_{1}^{2}}\left[A_{+}, A_{-}\right]
$$

and

$$
\partial_{-} B_{+}=-\frac{1-\lambda_{0}^{-1} \lambda_{2}}{1-\lambda_{2}^{2}}\left[B_{+}, B_{-}\right], \quad \partial_{+} B_{-}=\frac{1-\lambda_{0} \lambda_{2}}{1-\lambda_{2}^{2}}\left[B_{+}, B_{-}\right]
$$

We are now in a position to write down the Lax pairs which imply that the theory is integrable. The Lax pairs should satisfy the relations

$$
\partial_{+} \mathcal{L}_{-}^{(i)}-\partial_{-} \mathcal{L}_{+}^{(i)}=\left[\mathcal{L}_{+}^{(i)}, \mathcal{L}_{-}^{(i)}\right], \quad i=1,2
$$


where each of the Lax pairs $\mathcal{L}_{ \pm}^{(i)}\left(\tau, \sigma ; \zeta_{i}\right)$ will depend on a spectral parameter $\zeta_{i} \in \mathbb{C}$. Furthemore, for notational convenience we drop the subscript from $\zeta_{i}$. One can easily show that the Lax pair in the case of (3.8) is given by

$$
\mathcal{L}_{ \pm}=\frac{2 \zeta}{\zeta \mp 1} \tilde{A}_{ \pm}, \quad \tilde{A}_{+}=\frac{1-\lambda_{0}^{-1} \lambda_{1}}{1-\lambda_{1}^{2}} A_{+}, \quad \tilde{A}_{-}=\frac{1-\lambda_{0} \lambda_{1}}{1-\lambda_{1}^{2}} A_{-}, \quad \zeta \in \mathbb{C} .
$$

Similarly, for (3.9) the Lax pair is given by

$$
\mathcal{L}_{ \pm}=\frac{2 \zeta}{\zeta \mp 1} \tilde{B}_{ \pm}, \quad \tilde{B}_{+}=\frac{1-\lambda_{0} \lambda_{2}}{1-\lambda_{2}^{2}} B_{+}, \quad \tilde{B}_{-}=\frac{1-\lambda_{0}^{-1} \lambda_{2}}{1-\lambda_{2}^{2}} B_{-}, \quad \zeta \in \mathbb{C} .
$$

The careful reader may have noticed that our claim that the theory is integrable is not quite proven yet. One should in addition show that the conserved charges obtained from the two Lax pairs above are in involution. To this end we define dressed currents in such a way that when these are expressed in terms of canonical variables they have the same form as the corresponding currents of a WZW model. This idea was employed for the gauged WZW models [50] and in the present context in [44]. In particular, we define the dressed currents as

$$
\begin{array}{ll}
\mathcal{J}_{+}^{(1)}=D_{+} g_{1} g_{1}^{-1}+A_{+}-A_{-}, & \mathcal{J}_{-}^{(1)}=-g_{1}^{-1} D_{-} g_{1}+B_{-}-B_{+}, \\
\mathcal{J}_{+}^{(2)}=D_{+} g_{2} g_{2}^{-1}+B_{+}-B_{-}, & \mathcal{J}_{-}^{(2)}=-g_{2}^{-1} D_{-} g_{2}+A_{-}-A_{+} .
\end{array}
$$

These currents obey four independent commuting copies of current algebras [44]

$$
\left\{\mathcal{J}_{ \pm}^{(i) a}, \mathcal{J}_{ \pm}^{(i) b}\right\}=\frac{2}{k_{i}} f_{a b c} \mathcal{J}_{ \pm}^{(i) c} \delta_{\sigma \sigma^{\prime}} \pm \frac{2}{k_{i}} \delta_{a b} \delta_{\sigma \sigma^{\prime}}^{\prime}, \quad\left\{\mathcal{J}_{ \pm}^{(i) a}, \mathcal{J}_{\mp}^{(i) b}\right\}=0, \quad i=1,2
$$

which encode the canonical structure of the theory. Using the definitions (3.13), the constraints (3.1) and (3.2) can be written as

$$
\begin{array}{ll}
\mathcal{J}_{+}^{(1)}=\lambda_{0}^{-1} \lambda_{1}^{-T} A_{+}-A_{-}, & \mathcal{J}_{-}^{(1)}=\lambda_{0}^{-1} \lambda_{2}^{-1} B_{-}-B_{+}, \\
\mathcal{J}_{+}^{(2)}=\lambda_{0} \lambda_{2}^{-T} B_{+}-B_{-}, & \mathcal{J}_{-}^{(2)}=\lambda_{0} \lambda_{1}^{-1} A_{-}-A_{+} .
\end{array}
$$

These equations can be easily inverted in order to express the $A_{ \pm}$and $B_{ \pm}$in terms of the dressed currents. By just inspecting (3.15) it is easy to see that $A_{ \pm}$will depend only on $\mathcal{J}_{+}^{(1)}$ and $\mathcal{J}_{-}^{(2)}$ while $B_{ \pm}$will depend only on $\mathcal{J}_{-}^{(1)}$ and $\mathcal{J}_{+}^{(2)}$. Since the Poisson brackets of any of the variables in the set $\left\{\mathcal{J}_{+}^{(1)}, \mathcal{J}_{-}^{(2)}\right\}$ with any of the variables in the set $\left\{\mathcal{J}_{-}^{(1)}, \mathcal{J}_{+}^{(2)}\right\}$ is zero we conclude that the Poisson bracket between $A_{ \pm}$and $B_{ \pm}$is zero, that is $\left\{A_{ \pm}, B_{ \pm}\right\}$P.B. $=0$. This completes the proof that the charges generated by the Lax pair of (3.11) and those generated by the Lax pair of (3.12) are in involution. Thus, the theory defined by $(2.12)$ is integrable. ${ }^{1}$ It would be interesting to see if there are other choices of

\footnotetext{
${ }^{1}$ One might wonder if the conserved changes provided by each one of the Lax pairs are among themselves in involution due the non-ultralocal term proportional to $\delta^{\prime}$ in (3.14). Such terms give rise to non-ultralocal terms in the Poisson algebra of the $\mathcal{L}_{\sigma}$ which is used to define the monodromy matrix and from that to construct the infinite tower of conserved changes. Nevertheless, it has been shown in [51, 52] that the presence of such terms does not spoil the fact that the infinite number of conserved charges are in involution (eqs. (3.23) and (3.24) of [52]) provided that the Poisson brackets of $\mathcal{L}_{\sigma}$ assume the Maillet form and the modified Yang-Baxter equation is satisfied. Our effective action (2.12) implies a canonical structure which is precisely a double copy of the two-parameter deformation of the PCM's canonical structure presented in [53]. In this work it was shown that the Maillet brackets are satisfied and an explicit solution to the modified classical Yang-Baxter equation was found (see section 3, where the parameter $\rho$ in there is related to the level asymmetry as in eq. (4.4) of [43]).
} 
the deformation matrices $\lambda_{1}$ and $\lambda_{2}$ for which the theory (2.12) remains integrable. For the case of equal levels a classification of the different integrable cases was performed in [44] based on previous work for single $\lambda$-deformations $[1,2,14,15]$.

\section{The $\beta$-function}

One may attempt to use the $\sigma$-model background fields for (2.12) in order to compute using the renormalization group equations the beta-function equations for the parameters $\lambda_{i}, i=1,2$. This seems clearly a formidable task. However, the fact that in (2.17) the defining CFT theories, that is the two WZW models, are decoupled, implies that there is no mixing between the two deformation parameters. The arguments in favour of that are identical to those presented for the equal level case in [45]. In fact the beta-function found by CFT perturbative methods in [42] and in [43] is

$$
\frac{d \lambda}{d t}=-\frac{c_{G}}{2 \sqrt{k_{1} k_{2}}} \frac{\lambda^{2}\left(\lambda-\lambda_{0}\right)\left(\lambda-\lambda_{0}^{-1}\right)}{\left(1-\lambda^{2}\right)^{2}},
$$

where $t=\ln \mu^{2}$ with $\mu$ an energy scale and where $\lambda$ could be either $\lambda_{1}$ or $\lambda_{2}$. The above formula is valid for $k_{1}, k_{2} \gg 1$. Clearly, the RG flow is between $\lambda=0$ at the UV and the fixed point in the IR at $\lambda=\lambda_{0}$. The other fixed point at $\lambda_{0}^{-1}$ is unphysical since via the duality (2.14) it corresponds to a theory with negative levels. We also note that, even though we do not know the RG flows equations for general $\lambda_{a b}$, it is guaranteed that $\lambda_{a b}=\lambda_{0} \delta_{a b}$ is an IR fixed point.

Clearly, one should be able to compute the above by first setting one of these parameters to zero and then using the resulting action which is much simpler. ${ }^{2}$ For the equal level case, this approach was taken in [45] resulting into a complete agreement with the CFT results. In our case we will use the action (2.18) in order to compute the one loop RG flow equations [54-60]

$$
\frac{d}{d t}\left(G_{\mu \nu}+B_{\mu \nu}\right)=R_{\mu \nu}^{-}
$$

where the Ricci tensor includes the torsion. In this paper, we will do this exercise for the case of isotropic couplings $\lambda_{a b}=\lambda \delta_{a b}$.

The first step is to define the frames by writing the metric in the form

$$
d s^{2}=\frac{k_{2}}{2}\left(e^{a} e^{a}+e^{\hat{a}} e^{\hat{a}}\right),
$$

where

$$
e^{a}=\lambda_{0} \sqrt{1-\lambda^{2}} R^{a}, \quad e^{\hat{a}}=L^{\hat{a}}+\lambda_{0} \lambda R^{a} .
$$

We have disregarded a factor of $\frac{k_{2}}{2}$ in the definition of the frames which will be easily restored later and defined for notational convenience that $R_{1}^{a}=R^{a}$ and that $L_{2}^{a}=L^{\hat{a}}$.

We may compute all geometrical data using the relations

$$
d L^{a}=\frac{1}{2} f_{a b c} L^{b} \wedge L^{c}, \quad d R^{a}=-\frac{1}{2} f_{a b c} R^{b} \wedge R^{c} .
$$

\footnotetext{
${ }^{2}$ This can be consistently done since $\lambda_{2}=0$ is a UV fixed point of the RG flow equations.
} 
We also need the antisymmetric tensor. In a two-form notation and pulling out, as in the case for the metric the factor $\frac{k_{2}}{2}$, this is given by

$$
B=B_{0}+\lambda_{0} \lambda R^{a} \wedge L^{\hat{a}}
$$

where $B_{0}$ is the two-form corresponding to the two WZW models, so that $H_{0}=d B_{0}$. Using the frames defined in (4.4) we have that

$$
\begin{aligned}
H_{0}=-\frac{1}{6} f_{a b c}\left(\lambda_{0}^{2} R^{a} \wedge R^{b} \wedge R^{c}+L^{\hat{a}} \wedge L^{\hat{b}} \wedge L^{\hat{c}}\right) \\
=f_{a b c}\left(-\frac{1-\lambda_{0} \lambda^{3}}{6 \lambda_{0}\left(1-\lambda^{2}\right)^{3 / 2}} e^{a} \wedge e^{b} \wedge e^{c}-\frac{1}{6} e^{\hat{a}} \wedge e^{\hat{b}} \wedge e^{\hat{c}}\right. \\
\left.\quad-\frac{1}{2} \frac{\lambda^{2}}{1-\lambda^{2}} e^{\hat{a}} \wedge e^{b} \wedge e^{c}+\frac{1}{2} \frac{\lambda}{\sqrt{1-\lambda^{2}}} e^{a} \wedge e^{\hat{b}} \wedge e^{\hat{c}}\right) .
\end{aligned}
$$

In addition, the interaction term induces the following contribution to the three-form

$$
\begin{aligned}
H_{\lambda} & =\lambda_{0} \lambda d\left(R^{a} \wedge L^{\hat{a}}\right)=-\frac{\lambda_{0}}{2} \lambda f^{a b c} R^{a} \wedge L^{\hat{b}} \wedge\left(L^{\hat{c}}+R^{c}\right) \\
& =\frac{\lambda}{2 \sqrt{1-\lambda^{2}}} f^{a b c}\left(\frac{\lambda\left(1-\lambda_{0} \lambda\right)}{\lambda_{0}\left(1-\lambda^{2}\right)} e^{a} \wedge e^{b} \wedge e^{c}-e^{\hat{a}} \wedge e^{\hat{b}} \wedge e^{c}+\frac{2 \lambda_{0} \lambda-1}{\lambda_{0} \sqrt{1-\lambda^{2}}} e^{\hat{a}} \wedge e^{b} \wedge e^{c}\right) .
\end{aligned}
$$

As a result the field strength of the $B$-field reads

$$
\begin{aligned}
H=d B=H_{0}+H_{\lambda}=-\frac{1}{6} f_{a b c}( & \frac{1-3 \lambda^{2}+2 \lambda_{0} \lambda^{3}}{\lambda_{0}\left(1-\lambda^{2}\right)^{3 / 2}} e^{a} \wedge e^{b} \wedge e^{c} \\
& \left.+e^{\hat{a}} \wedge e^{\hat{b}} \wedge e^{\hat{c}}+\frac{3 \lambda\left(1-\lambda_{0} \lambda\right)}{\lambda_{0}\left(1-\lambda^{2}\right)} e^{\hat{a}} \wedge e^{b} \wedge e^{c}\right) .
\end{aligned}
$$

From the last equation it is straightforward to read off the components

$$
H_{a b c}=-\frac{1-3 \lambda^{2}+2 \lambda_{0} \lambda^{3}}{\lambda_{0}\left(1-\lambda^{2}\right)^{3 / 2}} f_{a b c}, \quad H_{\hat{a} \hat{b} \hat{c}}=-f_{a b c}, \quad H_{\hat{a} b c}=-\frac{\lambda\left(1-\lambda_{0} \lambda\right)}{\lambda_{0}\left(1-\lambda^{2}\right)} f_{a b c} .
$$

In a double index notation $A=(a, \hat{a})$ the geometric data can be found using the relation

$$
d e^{A}+\omega_{B}^{A} \wedge e^{B}=0 .
$$

From this ones extracts the spin connection one-form $\omega^{A B}$ and finds that

$$
\begin{aligned}
& \omega^{a b}=f_{a b c}\left(\omega_{1} e^{c}+\omega_{2} e^{\hat{c}}\right), \\
& \omega^{a \hat{b}}=\omega^{\hat{a} b}=f_{a b c}\left(\omega_{3} e^{c}+\omega_{4} e^{\hat{c}}\right), \\
& \omega^{\hat{a} \hat{b}}=f_{a b c}\left(\omega_{5} e^{c}+\omega_{6} e^{\hat{c}}\right),
\end{aligned}
$$

where

$$
\begin{array}{ll}
\omega_{1}=-\frac{1}{2 \lambda_{0} \sqrt{1-\lambda^{2}}}, & \omega_{2}=\frac{\lambda\left(1-\lambda_{0} \lambda\right)}{2 \lambda_{0}\left(1-\lambda^{2}\right)}, \\
\omega_{3}=-\frac{\lambda\left(1-\lambda_{0} \lambda\right)}{2 \lambda_{0}\left(1-\lambda^{2}\right)}, & \omega_{4}=0, \\
\omega_{5}=-\frac{\lambda}{\sqrt{1-\lambda^{2}}}, & \omega_{6}=\frac{1}{2} .
\end{array}
$$


It is convenient for our purposes to use the spin connection with torsion. This is defined as

$$
\omega_{-}^{A B}=\omega^{A B}-\frac{1}{2} H^{A B} e^{C} .
$$

The components of the torsionfull spin connection read

$$
\begin{aligned}
& \omega_{-}^{a b}=f_{a b c}\left(c_{1} e^{c}+c_{2} e^{\hat{c}}\right), \\
& \omega_{-}^{\hat{a} \hat{b}}=f_{a b c}\left(\hat{c}_{1} e^{c}+\hat{c}_{2} e^{\hat{c}}\right), \\
& \omega_{-}^{a \hat{b}}=\omega_{-}^{\hat{a} b}=0,
\end{aligned}
$$

where

$$
\begin{array}{ll}
c_{1}=-\frac{\lambda^{2}\left(1-\lambda_{0} \lambda\right)}{\lambda_{0}\left(1-\lambda^{2}\right)^{3 / 2}}, & c_{2}=\frac{\lambda\left(1-\lambda_{0} \lambda\right)}{\lambda_{0}\left(1-\lambda^{2}\right)}, \\
\hat{c}_{1}=-\frac{\lambda}{\left(1-\lambda^{2}\right)^{1 / 2}}, & \hat{c}_{2}=1 .
\end{array}
$$

Then we proceed to compute the generalized Riemann tensor defined by

$$
R_{-}^{A B}=\frac{1}{2} R_{-}^{A B}{ }_{C D} e^{C} \wedge e^{D}=d \omega_{-}^{A B}+\omega_{-}^{A C} \wedge \omega_{-}^{C B} .
$$

In our case this takes the form

$$
\begin{aligned}
& R_{-}^{a b}=d \omega_{-}^{a b}+\omega_{-}^{a c} \wedge \omega_{-}^{c b}, \\
& R_{-}^{a \hat{b}}=0, \\
& R_{-}^{\hat{a} \hat{b}}=d \omega_{-}^{\hat{a} \hat{b}}+\omega_{-}^{\hat{a} \hat{c}} \wedge \omega_{-}^{\hat{c} \hat{b}} .
\end{aligned}
$$

As a result, the components the generalized Riemann tensor reads

$$
\begin{array}{ll}
R_{-d e}^{a b}=R_{1} f_{a b c} f_{c d e}, & R_{1}=2 c_{1} \omega_{1}+2 c_{2} \omega_{3}-c_{1}^{2}, \\
R_{-d \hat{e}}^{a b}=R_{2} f_{a b c} f_{c d e}, & R_{2}=c_{1} \omega_{2}+c_{1} \omega_{3}+c_{2} \omega_{5}+c_{2} \omega_{4}-c_{1} c_{2}, \\
R_{-\hat{d} \hat{e}}^{a b}=R_{3} f_{a b c} f_{c d e}, & R_{3}=2 c_{1} \omega_{4}+2 c_{2} \omega_{6}-c_{2}^{2} .
\end{array}
$$

The components of $R_{-}^{\hat{a} \hat{b}} A B$ are obtained by simply replacing $c_{i}$ by $\hat{c}_{i}$, leading to

$$
\begin{array}{ll}
R_{-d e}^{\hat{a} \hat{b}}=\hat{R}_{1} f_{a b c} f_{c d e}, & \hat{R}_{1}=2 \hat{c}_{1} \omega_{1}+2 \hat{c}_{2} \omega_{3}-\hat{c}_{1}^{2}, \\
R_{-d \hat{e}}^{\hat{a} \hat{b}}=\hat{R}_{2} f_{a b c} f_{c d e}, & \hat{R}_{2}=\hat{c}_{1} \omega_{2}+\hat{c}_{1} \omega_{3}+\hat{c}_{2} \omega_{5}+\hat{c}_{2} \omega_{4}-\hat{c}_{1} \hat{c}_{2}, \\
R_{-}^{\hat{a} \hat{b} \hat{d} \hat{e}}=\hat{R}_{3} f_{a b c} f_{c d e}, & \hat{R}_{3}=2 \hat{c}_{1} \omega_{4}+2 \hat{c}_{2} \omega_{6}-\hat{c}_{2}^{2} .
\end{array}
$$

Using the definition of the Ricci tensor $R_{-}^{A B}=R_{-}^{A C}{ }_{B C}$ we find that

$$
\begin{array}{ll}
R_{-}^{a b}=c_{G} \delta_{a b} R_{1}, & R_{-}^{a \hat{b}}=c_{G} \delta_{a b} R_{2}, \\
R_{-}^{\hat{a} b}=c_{G} \delta_{a b} \hat{R}_{2}, & R_{-}^{\hat{a} \hat{b}}=c_{G} \delta_{a b} \hat{R}_{1} .
\end{array}
$$


Since the frame as defined in (4.4) depends on $\lambda$, we convert to the $R^{a}, L^{\hat{a}}$ basis. We may change basis components using

$$
\begin{aligned}
R_{-}^{a b} e_{+}^{a} e_{-}^{b} & +R_{-}^{a \hat{b}} e_{+}^{a} e_{-}^{\hat{b}}+R_{-}^{\hat{a} b} e_{+}^{\hat{a}} e_{-}^{b}+R_{-}^{\hat{a} \hat{b}} e_{+}^{\hat{a}} e_{-}^{\hat{b}} \\
& =\tilde{R}_{-}^{a b} R_{+}^{a} R_{-}^{b}+\tilde{R}_{-}^{\hat{a} \hat{b}} L_{+}^{\hat{a}} L_{-}^{\hat{b}}+\tilde{R}_{-}^{a \hat{b}} R_{+}^{a} L_{-}^{\hat{b}}+\tilde{R}_{-}^{\hat{a} b} L_{+}^{\hat{a}} R_{-}^{b}
\end{aligned}
$$

where \pm as subscripts denote the corresponding light-cone versions of the frames where the exterior derivative is replaced by the worldsheet derivatives. We have also used tilded symbols for the components in the $R^{a}, L^{\hat{a}}$ basis. Then we find that

$$
\begin{aligned}
& \tilde{R}_{-}^{a b}=\lambda_{0}^{2}\left(1-\lambda^{2}\right) R_{-}^{a b}+\lambda_{0}^{2} \lambda \sqrt{1-\lambda^{2}}\left(R_{-}^{a \hat{b}}+R_{-}^{\hat{a} b}\right)+\lambda_{0}^{2} \lambda^{2} R_{-}^{\hat{a} \hat{b}}, \\
& \tilde{R}_{-}^{\hat{a} \hat{b}}=R_{-}^{\hat{a} \hat{b}} \\
& \tilde{R}_{-}^{a \hat{b}}=\lambda_{0} \sqrt{1-\lambda^{2}} R_{-}^{a \hat{b}}+\lambda_{0} \lambda R_{-}^{\hat{a} \hat{b}}, \\
& \tilde{R}_{-}^{\hat{a} b}=\lambda_{0} \sqrt{1-\lambda^{2}} R_{-}^{\hat{a} b}+\lambda_{0} \lambda R_{-}^{\hat{a} \hat{b}} .
\end{aligned}
$$

The above procedure applies for any tensor replacing the Ricci tensor $R_{-}^{A B}$. By using (4.21), we obtain that

$$
\begin{aligned}
& \tilde{R}_{-}^{a b}=c_{G} \lambda_{0}^{2} \delta_{a b}\left(\left(1-\lambda^{2}\right) R_{1}+\lambda \sqrt{1-\lambda^{2}}\left(R_{2}+\hat{R}_{2}\right)+\lambda^{2} \hat{R}_{1}\right), \\
& \tilde{R}_{-}^{\hat{a} \hat{b}}=c_{G} \delta_{a b} \hat{R}_{1} \\
& \tilde{R}_{-}^{a \hat{b}}=c_{G} \lambda_{0} \delta_{a b}\left(\sqrt{1-\lambda^{2}} R_{2}+\lambda \hat{R}_{1}\right), \\
& \tilde{R}_{-}^{\hat{a} b}=c_{G} \lambda_{0} \delta_{a b}\left(\sqrt{1-\lambda^{2}} \hat{R}_{2}+\lambda \hat{R}_{1}\right) .
\end{aligned}
$$

Specifically, the various coefficients are given by

$$
\begin{array}{ll}
R_{1}=\frac{\lambda^{3}\left(1-\lambda_{0} \lambda\right)\left(\lambda_{0}-\lambda\right)}{\lambda_{0}^{2}\left(1-\lambda^{2}\right)^{3}}, & R_{2}=-\frac{\lambda^{2}\left(1-\lambda_{0} \lambda\right)\left(\lambda_{0}-\lambda\right)}{\lambda_{0}^{2}\left(1-\lambda^{2}\right)^{5 / 2}}, \\
R_{3}=\frac{\lambda\left(1-\lambda_{0} \lambda\right)\left(\lambda_{0}-\lambda\right)}{\lambda_{0}^{2}\left(1-\lambda^{2}\right)^{2}}, & \hat{R}_{1}=\hat{R}_{2}=\hat{R}_{3}=0,
\end{array}
$$

from which we find that

$$
\tilde{R}_{-}^{a \hat{b}}=-c_{G} \delta_{a b} \frac{\lambda^{2}\left(\lambda-\lambda_{0}\right)\left(\lambda-\lambda_{0}^{-1}\right)}{\left(1-\lambda^{2}\right)^{2}}
$$

All other components of the Ricci tensor vanish. After restoring the overall factor of $\frac{k_{2}}{2}$, equation (4.2) gives for the running of the coupling $\lambda$ the same expression as the one in (4.1).

\section{Symmetry at the IR conformal point}

Our models provide an explicit example of an integrable smooth flow between exact CFTs. One of the end points is the sum of two WZW models at different levels. In this section we investigate the other end point, that is the nature and the symmetries of the CFTs to which the theory flows as one approaches the IR regime. We will consider the two cases $\left(\lambda_{1}, \lambda_{2}\right)=\left(\lambda_{0}, \lambda_{0}\right)$ and $\left(\lambda_{1}, \lambda_{2}\right)=\left(\lambda_{0}, 0\right)$ separately. In each case we will specify the corresponding CFT and its symmetries. 


\subsection{CFT and its symmetries at $\lambda_{1}=\lambda_{2}=\lambda_{0}$}

The corresponding CFT is obtained by setting $\lambda_{1}=\lambda_{2}=\lambda_{0} \delta_{a b}$ in (2.12). However, the symmetries of the CFT are more clearly exhibited if one uses the action before integrating out the fields $A_{ \pm}$and $B_{ \pm}$. In this case the action (2.6) becomes

$$
\begin{aligned}
S= & S_{k_{1}}\left(g_{1}\right)+S_{k_{2}}\left(g_{2}\right)+\frac{k_{1}}{\pi} \int d^{2} \sigma \operatorname{Tr}\left(A_{-} \partial_{+} g_{1} g_{1}^{-1}-B_{+} g_{1}^{-1} \partial_{-} g_{1}+A_{-} g_{1} B_{+} g_{1}^{-1}\right) \\
& +\frac{k_{2}}{\pi} \int d^{2} \sigma \operatorname{Tr}\left(B_{-} \partial_{+} g_{2} g_{2}^{-1}-A_{+} g_{2}^{-1} \partial_{-} g_{2}+B_{-} g_{2} A_{+} g_{2}^{-1}-A_{+} A_{-}-B_{+} B_{-}\right) .
\end{aligned}
$$

In order to clarify the nature of this CFT consider the following infinitesimal transformations

$$
\begin{array}{rlrl}
\delta A_{ \pm} & =-\partial_{ \pm} u_{L}+\left[A_{ \pm}, u_{L}\right], \quad \delta B_{ \pm} & =-\partial_{ \pm} u_{R}+\left[B_{ \pm}, u_{R}\right], \\
\delta g_{1} & =-u_{L} g_{1}+g_{1} u_{R}, & \delta g_{2} & =-u_{R} g_{2}+g_{2} u_{L} .
\end{array}
$$

Then the variation of the action at the IR fixed point becomes

$$
\delta S=\frac{k_{2}-k_{1}}{\pi} \int d^{2} \sigma \operatorname{Tr}\left(A_{-} \partial_{+} u_{L}+B_{+} \partial_{-} u_{R}\right) .
$$

Hence, if $u_{L}=u_{L}\left(\sigma^{-}\right)$and $u_{R}=u_{R}\left(\sigma^{+}\right)$, the action remains invariant. We may investigate this in more detail by writing the would-be gauge fields $A_{ \pm}$and $B_{ \pm}$as

$$
A_{ \pm}=\partial_{ \pm} h_{ \pm} h_{ \pm}^{-1}, \quad B_{ \pm}=\partial_{ \pm} k_{ \pm} k_{ \pm}^{-1}, \quad h_{ \pm}, k_{ \pm} \in G .
$$

The finite version of the transformation (5.2) for the $A_{ \pm}$and $B_{ \pm}$is equivalent to the following transformation for $h$ and $k$, namely $h_{ \pm} \rightarrow L^{-1} h_{ \pm}$and $k_{ \pm} \rightarrow R^{-1} k_{ \pm}$. Then, with the aid of the Polyakov-Wiegmann formula we may write the action (5.1) as

$$
\begin{aligned}
S= & S_{k_{1}}\left(h_{-}^{-1} g_{1} k_{+}\right)+S_{k_{2}-k_{1}}\left(k_{-}^{-1} g_{2} h_{+}\right)-S_{k_{2}}\left(h_{-}^{-1} h_{+}\right) \\
& +S_{k_{1}}\left(k_{-}^{-1} g_{2} h_{+}\right)-S_{k_{2}}\left(k_{-}^{-1} k_{+}\right) \\
& +S_{k_{2}-k_{1}}\left(h_{-}^{-1}\right)+S_{k_{2}-k_{1}}\left(k_{+}\right) .
\end{aligned}
$$

The symmetry group of the CFT becomes transparent if we change variables as $g_{1} \rightarrow$ $g_{1} h_{+} k_{+}^{-1}$ and $g_{2} \rightarrow k_{-} h_{-}^{-1} g_{2}$. Then the action can be cast as

$$
\begin{aligned}
S= & S_{k_{1}}\left(h_{-}^{-1} g_{1} h_{+}\right)+S_{k_{2}-k_{1}}\left(h_{-}^{-1} g_{2} h_{+}\right)-S_{k_{2}}\left(h_{-}^{-1} h_{+}\right) \\
& +S_{k_{1}}\left(h_{-}^{-1} g_{2} h_{+}\right)-S_{k_{2}}\left(k_{-}^{-1} k_{+}\right) \\
& +S_{k_{2}-k_{1}}\left(h_{-}^{-1}\right)+S_{k_{2}-k_{1}}\left(k_{+}\right) .
\end{aligned}
$$

The first line is the gauged WZW action for the coset CFT

$$
\left.\left.\frac{G_{k_{1}} \times G_{k_{2}-k_{1}}}{G_{k_{2}}}\right|_{L} \otimes \frac{G_{k_{1}} \times G_{k_{2}-k_{1}}}{G_{k_{2}}}\right|_{R},
$$

indicating both the left and the right sectors. The conformal invariance is generated by the transformations

$$
\begin{array}{ll}
h_{+} \rightarrow h_{+} \Omega\left(\sigma^{-}\right), & k_{+} \rightarrow k_{+} \Omega\left(\sigma^{-}\right), \\
h_{-} \rightarrow h_{-} \tilde{\Omega}\left(\sigma^{+}\right), & k_{-} \rightarrow k_{-} \tilde{\Omega}\left(\sigma^{+}\right) .
\end{array}
$$


Under these the second and third lines of (5.6) generate two copies of the current algebra for $G$ for the left and the right movers, but at level zero. Hence, for unitary representations this is trivial.

The action (5.6) is also invariant under

$$
\begin{array}{rlrl}
h_{ \pm} & \rightarrow L^{-1}\left(\sigma^{-}\right) h_{ \pm}, & k_{ \pm} & \rightarrow R^{-1}\left(\sigma^{+}\right) k_{ \pm}, \\
g_{1} & \rightarrow L^{-1}\left(\sigma^{-}\right) g_{1} L\left(\sigma^{-}\right), \quad g_{2} & \rightarrow L^{-1}\left(\sigma^{-}\right) g_{2} L\left(\sigma^{-}\right) .
\end{array}
$$

This generates the current algebra theory

$$
\left.\left.G_{k_{2}-k_{1}}\right|_{L} \otimes G_{k_{2}-k_{1}}\right|_{R}
$$

Combining the above and (5.7) we obtain the following flow of CFTs from the UV at $\lambda_{1}=\lambda_{2}=0$ towards the IR at $\lambda_{1}=\lambda_{2}=\lambda_{0}$

$$
G_{k_{1}} \times G_{k_{2}} \stackrel{\mathrm{IR}}{\Longrightarrow} \frac{G_{k_{1}} \times G_{k_{2}-k_{1}}}{G_{k_{2}}} \times G_{k_{2}-k_{1}},
$$

one copy for the left and an identical one for the right movers. This flow was speculated for the $\mathrm{SU}(2)$ case in [61] based mainly on symmetry arguments and further supported in [43] for general groups based on the form of the anomalous dimensions of the current operators in the CFT point in the IR. Note that this flow is in accordance with Zamolodchikov's $c$-theorem [62] since the central charge in the IR is smaller than that in the UV.

\subsection{CFT and its symmetries at $\lambda_{1}=\lambda_{0}, \lambda_{2}=0$}

For the case of $\lambda_{2}=0$ the action (2.12) or equivalently (2.18) can be rewritten at the fixed point $\lambda_{a b}=\lambda_{0} \delta_{a b}$, by the use of the Polyakov-Wiegman identity as

$$
S=S_{k_{1}}\left(g_{2} g_{1}\right)+S_{k_{2}-k_{1}}\left(g_{2}\right) .
$$

This is the sum of two WZW actions with two copies of direct current algebra $G_{k_{1}} \times G_{k_{2}-k_{1}}$ for the left and the right movers. Hence, in this case, the theory smoothly flows from a CFT in the UV which is the sum of two WZW models, one with level $k_{1}$ and the other with level $k_{2}$ to another CFT in the IR which is the sum of two WZW models, at levels $k_{1}$ and $k_{2}-k_{1}$, respectively. That is

$$
G_{k_{1}} \times G_{k_{2}} \stackrel{\mathrm{IR}}{\Longrightarrow} G_{k_{1}} \times G_{k_{2}-k_{1}}
$$

one copy for the left and an identical one for the right movers. The IR theory has indeed a smaller central change that the one in the UV, again in accordance with Zamolodchikov's $c$-theorem.

One might worry that our conclusion for the CFTs (5.11) and (5.13) at the IR fixed point could be an artifact of the large level approximation of our analysis. However, the given answer in terms of exact CFTs leaves no doubt that the IR CFTs are the ones presented above but for finite level values. The form of $\lambda_{0}$ in (2.11) for finite values of the levels may change, but not its very existence.

Our discussion was valid as long as $\lambda_{0}<1$. When $\lambda_{0}=1$, i.e. $k_{1}=k_{2}$, then the IR fixed point seizes to exist and in fact the theory makes sense as long as we take an non-Abelian type limit [46] of the PCM for $G \times G$. 


\section{Discussions and future directions}

One of the intriguing features of two dimensional models is the existence of integrable quantum field theories interpolating between exact CFTs. The first example of such a flow was discovered in [63-65] and was realized via relevant perturbations of the unitary minimal models $\mathcal{M}_{p}$. For one sign of the coupling constant the theory was argued to flow to another minimal model, namely $\mathcal{M}_{p-1}$. Subsequently, these flows were generalized, by applying thermodynamic Bethe ansatz techniques, to more general unitary minimal models involving coset spaces [66-69] as well as to integrable flows between non-unitary theories of the type $\mathcal{M}_{p, q}[70,71]$ (see also [72-74]). However, in all the aforementioned examples the description was based on integrability arguments and the theories were lacking a Lagrangian formulation. In this paper, we explicitly constructed families of integrable $\sigma$-model actions smoothly interpolating between exact CFTs. Our realization uses two group elements of a general semi-simple group and may have two or one distinct deformation matrices.

Our construction resembles the similar construction of the doubly deformed integrable $\sigma$-models presented in [44] after allowing different levels for each of the WZW models. Despite the fact that the methods of construction are similar, making the levels different has major implications for the quantum behavior of the models. We have computed the $\beta$-function using gravitational methods and found that it exhibits a fixed point in the IR making our models particularly attractive. Moreover, we proved the remarkable fact that the resulting theories are integrable in the case of isotropic couplings. We explicitly constructed the Lax pairs and showed that the conserved charges are in involution.

Our models provide an explicit example of an integrable smooth flow between exact CFTs. At the UV point the theory is described by the sum of two WZW models, one at level $k_{1}$ and the other at level $k_{2}$. As soon as the perturbation is turned on our theories are driven towards another fixed point in the IR. When both coupling matrices are present, the IR CFT is described by a coset CFT whose symmetry group is given by (5.11). In the case of one coupling matrix the IR CFT is the sum of two WZW models one at level $k_{1}$ and the other at level $k_{2}-k_{1},(5.13)$. In both cases, the flow respects Zamolodchikov's $c$-theorem.

The motivation for the present paper was to find an action realizing all loop effects of the theory (1.2) including the existense of an IR fixed point. This goal has been achieved since the $\beta$-functions of our models do reproduce the all-loop $\beta$-function of the deformation of the left-right asymmetric CFTs which was previously derived in [42] and in [43] using CFT methods and non-perturbative symmetries of the theory. Although our realisation as a whole is left-right symmetric, as discussed below (2.17), our models reproduce accurately not only the exact $\beta$-function but also all the correlation functions of the left-right asymmetric model of (1.2).

A number of important questions still remains to be answered. It would be interesting to examine if one can construct a Lagrangian realization of the left-right asymmetric theories utilising a single group element and not two as we did in our construction. It would also be important to examine if the theories we constructed in this work can be embedded as solutions of type-II supergravity. Since our theories are integrable this may result to new integrable deformations of the $A d S_{5} \times S^{5}$ superstring, for example. Along the same 
lines, it would be interesting to see if there are other choices for the deformation matrices, except the isotropic one, that preserve the integrability of the model. Moreover, the fact that the $\lambda$ and $\eta$-deformations are related via Poisson-Lie T- duality and appropriate analytic continuations raises the question on the existence or not of new integrable models of the $\eta$-type which are dual to those constructed in the present work. Finally, it would be interesting to analyze the implications of our Lagrangian description in the context of chiral liquids in one dimension [61] since such systems should be apparently described by left-right asymmetric theories.

\section{Acknowledgments}

We acknowledge very useful discussions with K. Siampos. G. Georgiou would like to thank the Physics Department of the National and Kapodistrian U. of Athens for hospitality during this project.

Open Access. This article is distributed under the terms of the Creative Commons Attribution License (CC-BY 4.0), which permits any use, distribution and reproduction in any medium, provided the original author(s) and source are credited.

\section{References}

[1] K. Sfetsos, Integrable interpolations: from exact CFTs to non-Abelian T-duals, Nucl. Phys. B 880 (2014) 225 [arXiv:1312.4560] [InSPIRE].

[2] T.J. Hollowood, J.L. Miramontes and D.M. Schmidtt, Integrable deformations of strings on symmetric spaces, JHEP 11 (2014) 009 [arXiv: 1407.2840] [INSPIRE].

[3] T.J. Hollowood, J.L. Miramontes and D.M. Schmidtt, An integrable deformation of the $A d S_{5} \times S^{5}$ superstring, J. Phys. A 47 (2014) 495402 [arXiv: 1409.1538] [INSPIRE].

[4] G. Itsios, K. Sfetsos and K. Siampos, The all-loop non-Abelian Thirring model and its RG flow, Phys. Lett. B 733 (2014) 265 [arXiv:1404.3748] [INSPIRE].

[5] K. Sfetsos and K. Siampos, Gauged WZW-type theories and the all-loop anisotropic non-Abelian Thirring model, Nucl. Phys. B 885 (2014) 583 [arXiv:1405.7803] [INSPIRE].

[6] D. Kutasov, String theory and the non-Abelian Thirring model, Phys. Lett. B 227 (1989) 68 [INSPIRE].

[7] B. Gerganov, A. LeClair and M. Moriconi, On the $\beta$-function for anisotropic current interactions in 2D, Phys. Rev. Lett. 86 (2001) 4753 [hep-th/0011189] [INSPIRE].

[8] C. Appadu and T.J. Hollowood, $\beta$-function of $k$ deformed $A d S_{5} \times S^{5}$ string theory, JHEP 11 (2015) 095 [arXiv: 1507.05420] [INSPIRE].

[9] K. Sfetsos and D.C. Thompson, Spacetimes for $\lambda$-deformations, JHEP 12 (2014) 164 [arXiv: 1410.1886] [INSPIRE].

[10] S. Demulder, K. Sfetsos and D.C. Thompson, Integrable $\lambda$-deformations: squashing coset CFTs and $A d S_{5} \times S^{5}$, JHEP 07 (2015) 019 [arXiv: 1504.02781] [InSPIRE].

[11] R. Borsato, A.A. Tseytlin and L. Wulff, Supergravity background of $\lambda$-deformed model for $A d S_{2} \times S^{2}$ supercoset, Nucl. Phys. B 905 (2016) 264 [arXiv:1601.08192] [InSPIRE]. 
[12] Y. Chervonyi and O. Lunin, Supergravity background of the $\lambda$-deformed $A d S_{3} \times S^{3}$ supercoset, Nucl. Phys. B 910 (2016) 685 [arXiv:1606.00394] [INSPIRE].

[13] Y. Chervonyi and O. Lunin, Generalized $\lambda$-deformations of $A d S_{p} \times S^{p}$, Nucl. Phys. B 913 (2016) 912 [arXiv:1608.06641] [InSPIRE].

[14] K. Sfetsos and K. Siampos, The anisotropic $\lambda$-deformed $\mathrm{SU}(2)$ model is integrable, Phys. Lett. B 743 (2015) 160 [arXiv:1412.5181] [INSPIRE].

[15] K. Sfetsos, K. Siampos and D.C. Thompson, Generalised integrable $\lambda$ - and $\eta$-deformations and their relation, Nucl. Phys. B 899 (2015) 489 [arXiv: 1506.05784] [InSPIRE].

[16] C. Klimčík, Yang-Baxter $\sigma$-models and dS/AdS T duality, JHEP 12 (2002) 051 [hep-th/0210095] [INSPIRE].

[17] C. Klimčík, On integrability of the Yang-Baxter $\sigma$-model, J. Math. Phys. 50 (2009) 043508 [arXiv:0802.3518] [INSPIRE].

[18] C. Klimčík, Integrability of the bi-Yang-Baxter $\sigma$-model, Lett. Math. Phys. 104 (2014) 1095 [arXiv: 1402.2105] [INSPIRE].

[19] F. Delduc, M. Magro and B. Vicedo, On classical q-deformations of integrable $\sigma$-models, JHEP 11 (2013) 192 [arXiv:1308.3581] [INSPIRE].

[20] F. Delduc, M. Magro and B. Vicedo, An integrable deformation of the $A d S_{5} \times S^{5}$ superstring action, Phys. Rev. Lett. 112 (2014) 051601 [arXiv: 1309.5850] [INSPIRE].

[21] G. Arutyunov, R. Borsato and S. Frolov, S-matrix for strings on $\eta$-deformed $A d S_{5} \times S^{5}$, JHEP 04 (2014) 002 [arXiv: 1312.3542] [INSPIRE].

[22] C. Klimčík and P. Ševera, Dual non-Abelian duality and the Drinfeld double, Phys. Lett. B 351 (1995) 455 [hep-th/9502122] [INSPIRE].

[23] B. Vicedo, Deformed integrable $\sigma$-models, classical R-matrices and classical exchange algebra on Drinfel'd doubles, J. Phys. A 48 (2015) 355203 [arXiv:1504.06303] [inSPIRE].

[24] B. Hoare and A.A. Tseytlin, On integrable deformations of superstring $\sigma$-models related to $A d S_{n} \times S^{n}$ supercosets, Nucl. Phys. B 897 (2015) 448 [arXiv: 1504.07213] [inSPIRE].

[25] C. Klimčík, $\eta$ and $\lambda$ deformations as E-models, Nucl. Phys. B 900 (2015) 259 [arXiv: 1508.05832] [INSPIRE].

[26] C. Klimčík, Poisson-Lie T-duals of the bi-Yang-Baxter models, Phys. Lett. B 760 (2016) 345 [arXiv: 1606.03016] [INSPIRE].

[27] G. Georgiou, K. Sfetsos and K. Siampos, All-loop anomalous dimensions in integrable $\lambda$-deformed $\sigma$-models, Nucl. Phys. B 901 (2015) 40 [arXiv:1509.02946] [INSPIRE].

[28] G. Georgiou, K. Sfetsos and K. Siampos, All-loop correlators of integrable $\lambda$-deformed o-models, Nucl. Phys. B 909 (2016) 360 [arXiv:1604.08212] [INSPIRE].

[29] D. Kutasov, Duality off the critical point in two-dimensional systems with non-Abelian symmetries, Phys. Lett. B 233 (1989) 369 [INSPIRE].

[30] S. Demulder, D. Dorigoni and D.C. Thompson, Resurgence in $\eta$-deformed principal chiral models, JHEP 07 (2016) 088 [arXiv: 1604.07851] [INSPIRE].

[31] B. Hoare and S.J. van Tongeren, On Jordanian deformations of $A d S_{5}$ and supergravity, J. Phys. A 49 (2016) 434006 [arXiv: 1605.03554] [INSPIRE]. 
[32] D. Orlando, S. Reffert, J.-I. Sakamoto and K. Yoshida, Generalized type IIB supergravity equations and non-Abelian classical r-matrices, J. Phys. A 49 (2016) 445403 [arXiv: 1607.00795] [INSPIRE].

[33] G. Arutyunov, M. Heinze and D. Medina-Rincon, Integrability of the $\eta$-deformed Neumann-Rosochatius model, J. Phys. A 50 (2017) 035401 [arXiv:1607.05190] [InSPIRE].

[34] D. Osten and S.J. van Tongeren, Abelian Yang-Baxter deformations and TsT transformations, Nucl. Phys. B 915 (2017) 184 [arXiv:1608.08504] [InSPIRE].

[35] B. Hoare and A.A. Tseytlin, Homogeneous Yang-Baxter deformations as non-Abelian duals of the $A d S_{5}$ б-model, J. Phys. A 49 (2016) 494001 [arXiv:1609.02550] [InSPIRE].

[36] S.J. van Tongeren, Almost Abelian twists and AdS/CFT, Phys. Lett. B 765 (2017) 344 [arXiv: 1610.05677] [INSPIRE].

[37] D.M. Schmidtt, Exploring the lambda model of the hybrid superstring, JHEP 10 (2016) 151 [arXiv: 1609.05330] [INSPIRE].

[38] T. Araujo, I. Bakhmatov, E.Ó. Colgáin, J. Sakamoto, M.M. Sheikh-Jabbari and K. Yoshida, Yang-Baxter $\sigma$-models, conformal twists and noncommutative Yang-Mills theory, Phys. Rev. D 95 (2017) 105006 [arXiv:1702.02861] [InSPIRE].

[39] C. Klimčík, Yang-Baxter $\sigma$-model with WZNW term as E-model, Phys. Lett. B 772 (2017) 725 [arXiv:1706.08912] [INSPIRE].

[40] C. Appadu, T.J. Hollowood, D. Price and D.C. Thompson, Yang Baxter and anisotropic sigma and lambda models, cyclic RG and exact S-matrices, JHEP 09 (2017) 035 [arXiv: 1706.05322] [INSPIRE].

[41] J. Balog, P. Forgacs, Z. Horvath and L. Palla, A new family of SU(2) symmetric integrable $\sigma$-models, Phys. Lett. B 324 (1994) 403 [hep-th/9307030] [InSPIRE].

[42] A. LeClair, Chiral stabilization of the renormalization group for flavor and color anisotropic current interactions, Phys. Lett. B 519 (2001) 183 [hep-th/0105092] [INSPIRE].

[43] G. Georgiou, K. Sfetsos and K. Siampos, $\lambda$-deformations of left-right asymmetric CFTs, Nucl. Phys. B 914 (2017) 623 [arXiv:1610.05314] [InSPIRE].

[44] G. Georgiou and K. Sfetsos, A new class of integrable deformations of CFTs, JHEP 03 (2017) 083 [arXiv:1612.05012] [INSPIRE].

[45] G. Georgiou, E. Sagkrioti, K. Sfetsos and K. Siampos, Quantum aspects of doubly deformed CFTs, Nucl. Phys. B 919 (2017) 504 [arXiv:1703.00462] [INSPIRE].

[46] G. Georgiou, K. Sfetsos and K. Siampos, Double and cyclic $\lambda$-deformations and their canonical equivalents, Phys. Lett. B 771 (2017) 576 [arXiv:1704.07834] [INSPIRE].

[47] E. Witten, On holomorphic factorization of WZW and coset models, Commun. Math. Phys. 144 (1992) 189 [InSPIRE].

[48] O.A. Solovev, Towards conversion of the space of Thirring models into the model space for groups, Phys. Lett. B 309 (1993) 275 [InSPIRE].

[49] C.M. Hull and O.A. Solovev, Conformal points and duality of non-Abelian Thirring models and interacting WZNW models, Nucl. Phys. B 459 (1996) 243 [hep-th/9503021] [INSPIRE].

[50] P. Bowcock, Canonical quantization of the gauged Wess-Zumino model, Nucl. Phys. B 316 (1989) 80 [InSPIRE]. 
[51] J.M. Maillet, New integrable canonical structures in two-dimensional models, Nucl. Phys. B 269 (1986) 54 [inSPIRE].

[52] J.M. Maillet, Hamiltonian structures for integrable classical theories from graded Kac-Moody algebras, Phys. Lett. B 167 (1986) 401 [INSPIRE].

[53] G. Itsios, K. Sfetsos, K. Siampos and A. Torrielli, The classical Yang-Baxter equation and the associated Yangian symmetry of gauged WZW-type theories, Nucl. Phys. B 889 (2014) 64 [arXiv:1409.0554] [INSPIRE].

[54] G. Ecker and J. Honerkamp, Application of invariant renormalization to the nonlinear chiral invariant pion Lagrangian in the one-loop approximation, Nucl. Phys. B 35 (1971) 481 [INSPIRE].

[55] J. Honerkamp, Chiral multiloops, Nucl. Phys. B 36 (1972) 130 [INSPIRE].

[56] D. Friedan, Nonlinear models in two epsilon dimensions, Phys. Rev. Lett. 45 (1980) 1057 [INSPIRE].

[57] D. Friedan, Nonlinear models in $2+\epsilon$ dimensions, Annals Phys. 163 (1985) 318 [INSPIRE].

[58] T.L. Curtright and C.K. Zachos, Geometry, topology and supersymmetry in nonlinear models, Phys. Rev. Lett. 53 (1984) 1799 [INSPIRE].

[59] E. Braaten, T.L. Curtright and C.K. Zachos, Torsion and geometrostasis in nonlinear o-models, Nucl. Phys. B 260 (1985) 630 [Erratum ibid. B 266 (1986) 748] [INSPIRE].

[60] B.E. Fridling and A.E.M. van de Ven, Renormalization of generalized two-dimensional nonlinear $\sigma$ models, Nucl. Phys. B 268 (1986) 719 [InSPIRE].

[61] N. Andrei, M.R. Douglas and A. Jerez, Chiral liquids in one dimension: a non-Fermi-liquid class of fixed points, Phys. Rev. B 58 (1998) 7619 [cond-mat/9803134].

[62] A.B. Zamolodchikov, Irreversibility of the flux of the renormalization group in a $2 D$ field theory, JETP Lett. 43 (1986) 730 [Pisma Zh. Eksp. Teor. Fiz. 43 (1986) 565] [INSPIRE].

[63] A.B. Zamolodchikov, Renormalization group and perturbation theory near fixed points in two-dimensional field theory, Sov. J. Nucl. Phys. 46 (1987) 1090 [Yad. Fiz. 46 (1987) 1819] [INSPIRE].

[64] A.B. Zamolodchikov, From tricritical Ising to critical Ising by thermodynamic Bethe ansatz, Nucl. Phys. B 358 (1991) 524 [inSPIRE].

[65] A.W.W. Ludwig and J.L. Cardy, Perturbative evaluation of the conformal anomaly at new critical points with applications to random systems, Nucl. Phys. B 285 (1987) 687 [INSPIRE].

[66] A.B. Zamolodchikov, TBA equations for integrable perturbed $\mathrm{SU}(2)_{k} \times \mathrm{SU}(2)_{l} / \mathrm{SU}(2)_{k+1}$ coset models, Nucl. Phys. B 366 (1991) 122 [INSPIRE].

[67] V.A. Fateev and A.B. Zamolodchikov, Integrable perturbations of $Z_{N}$ parafermion models and $O(3) \sigma$-model, Phys. Lett. B 271 (1991) 91 [INSPIRE].

[68] F. Ravanini, Thermodynamic Bethe ansatz for $G_{k} \times G_{l} / G_{k+l}$ coset models perturbed by their $\phi_{1,1, \text { Adj }}$ operator, Phys. Lett. B 282 (1992) 73 [hep-th/9202020] [INSPIRE].

[69] F. Ravanini, R. Tateo and A. Valleriani, Dynkin TBAs, Int. J. Mod. Phys. A 8 (1993) 1707 [hep-th/9207040] [INSPIRE].

[70] M. Lassig, New hierarchies of multicriticality in two-dimensional field theory, Phys. Lett. B 278 (1992) 439 [INSPIRE]. 
[71] C.-R. Ahn, RG flows of nonunitary minimal CFTs, Phys. Lett. B 294 (1992) 204 [hep-th/9202028] [INSPIRE].

[72] M.J. Martins, Renormalization group trajectories from resonance factorized $S$ matrices, Phys. Rev. Lett. 69 (1992) 2461 [hep-th/9205024] [INSPIRE].

[73] F. Ravanini, M. Stanishkov and R. Tateo, Integrable perturbations of CFT with complex parameter: the $M_{3 / 5}$ model and its generalizations, Int. J. Mod. Phys. A 11 (1996) 677 [hep-th/9411085] [INSPIRE].

[74] P. Dorey, C. Dunning and R. Tateo, New families of flows between two-dimensional conformal field theories, Nucl. Phys. B 578 (2000) 699 [hep-th/0001185] [INSPIRE]. 